\title{
Uni-pluriversidad: 20 años de evolución del concepto de Universidad
}

Uni-pluriversidad: 20 years of evolution of University concept

\author{
Gustavo Eduardo Toledo Lara* (D)
}

\section{Resumen}

\section{Tipo de artículo:}

informes de investigación y ensayos inéditos. Separata: Uni-pluriversidad 20 años.

Doi: 10.17533/udea.unipluri.20.3.03

\section{Cómo citar este artículo:}

Toledo Lara, G. E. (2021). Uni-pluriversidad: 20 años de evolución del concepto de Universidad. Uni-Pluriversidad, 20(3), 1-15. https://doi. org/10.17533/udea.unipluri.20.3.03

Recibido: 13-09-2020 / Aprobado: 02-02-2021
Se presenta una revisión sobre la evolución de la definición de Universidad a lo largo de 20 años de la revista Uni-pluriversidad. Para esto, se establecieron dos fases: 1) aproximación teórica sobre el concepto y tipos de Universidad y 2) revisión de un conjunto de artículos para identificar las tendencias que han acompañado la definición de Universidad expuestas por los investigadores. Desde una perspectiva analítica-interpretativa y asumiendo la teoría fundamentada, se revisaron 590 artículos y se seleccionaron 41 para el muestreo comparativo constante y el muestreo teórico, identificando elementos similares o diferentes. Se concluye que una sola definición de Universidad no existe, aunque, desde el año 2001, se han expuesto en la revista elementos definitorios que pueden caracterizar la Universidad a la luz del contexto y de su pertinencia social, sin dejar de lado sus funciones tradicionales dentro del espectro de instituciones dedicadas a la formación universitaria.

Palabras clave: Universidad; tipos de universidad; concepto de Universidad.

* Universidad Camilo José Cela - España.

E-mail: gustavotoledolara@gmail.com

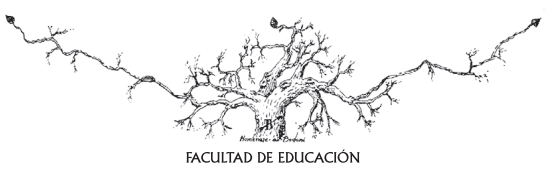




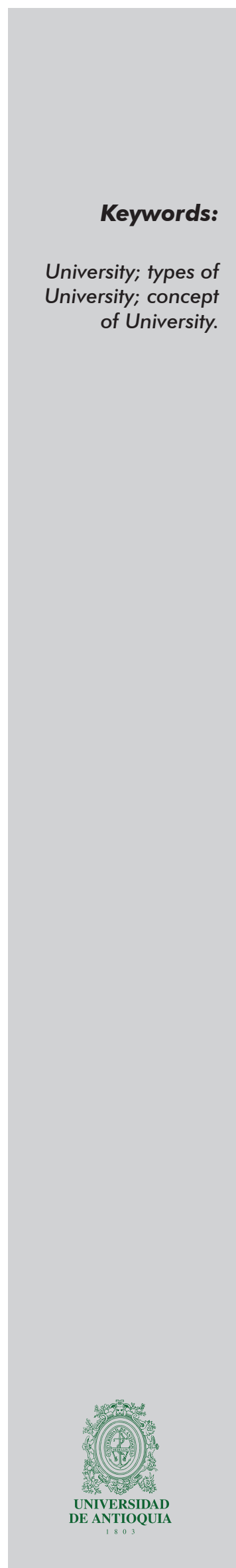

\section{Abstract}

A review on the evolution of the definition of University throughout 20 years of the journal Uni-pluriversidad is presented. For this purpose, two phases were established: 1) theoretical approach on concept and types of University, and 2) review of a set of articles to identify trends exposed by the researchers in relation to the definition of University. 590 articles were reviewed from an analytical-interpretative perspective and a grounded theory. Finally, 41 articles were selected for constant comparative sampling and a theoretical sampling, identifying similar or different aspects. It is concluded that there is no single definition of University, although since 2001, the journal has presented defining elements that can characterize University in the light of the context and its social relevance, without leaving aside its traditional functions within the spectrum of institutions dedicated to university education. 
Introducción

La Revista Uni-pluriversidad está de aniversario. Cumple 20 años desde su primera aparición en el contexto académico. Esto significa que a lo largo de todo el camino recorrido se han expuesto una serie de constataciones y consideraciones sobre el debate y la discusión que giran en torno al ser de la Universidad desde múltiples perspectivas. La observación de esta andadura es una oportunidad para el análisis de una institución que forma parte del quehacer humano desde hace muchos años y que ha experimentado los grandes influjos reformistas que el devenir histórico ha permitido percibir dentro del contexto universitario.

Así, desde el año 2001 se ha intentado construir una tribuna que sustente el debate en torno a la dinámica universitaria $y$, específicamente, sobre la razón de ser y la identidad de la Universidad como institución. En efecto, en todo este tiempo se han suscitado tensiones y transiciones que no han dejado indiferente a la Universidad. Cada momento permite la generación de lo que se entiende que debería ser la Universidad o más bien, del papel que esta ha de jugar en la sociedad. Hablamos entonces de una institución diversa, plural, incluyente, activa, viva, en constante movimiento y también en crisis.

También es de reconocer que la Universidad como institución ha tenido una vida con sus luces y sus sombras, es decir, esta dinámica de "altibajos" ha fomentado la búsqueda de aquella institución que sea lo más socialmente pertinente a la vez que lucha por seguir siendo Universidad.
Se trata así de una permanente tensión entre la tradición y el cambio, entre la racionalidad y la incertidumbre. En medio de este panorama, la revista Uni-pluriversidad ha sido testigo privilegiado del proceso por medio del cual, se le ha demandado a la Universidad las respuestas a todos los interrogantes de la humanidad mientras que hace ingentes esfuerzos por mantenerse sólida aun en los momentos más álgidos.

Desde una perspectiva cualitativa, el estudio que aquí se presenta tiene como objetivo principal presentar las tendencias que han sido expuestas en Uni-pluriversidad respecto al concepto de Universidad. Es decir, se exploran aquellos elementos clave que han definido el sentido de Universidad desde el año 2001 hasta el presente y cómo esto puede servir para observar la evolución de aquel concepto a lo largo de todos estos años para intentar determinar si, en efecto, este proceso evolutivo del concepto de Universidad ocurre de manera cíclica o de manera estática, con apenas cambios. Para llegar a este punto, se hace necesario superar dos fases: en una primera fase, se exploran las aportaciones relacionadas con las diversas posturas teóricas y conceptuales que, expresadas por medio de investigaciones científicas, muestran el estado del arte respecto a lo que conocemos como Universidad y sus tipos. En una segunda fase, se analizan las investigaciones presentadas desde Uni-pluriversidad para reconocer las tendencias que están presentes en el sentido de Universidad y cómo esto puede permitir inferir su concepto.

\section{Desarrollo}

\section{Universidad, concepto y tipos. Una aproximación teórica}

Hablar de Universidad no deja de ser todo un reto. Intentar definirla es aún más complejo, ya 
mos ciudadanos esperan mucho de ella, o porque en el propio seno de la Universidad el debate sobre su sentido y misión siempre está en permanente revisión y crítica a la luz del contexto en el que esta se encuentre. A propósito del contexto que rodea a la Universidad, se identifican ciertos rasgos que rodean el quehacer universitario y que, en su momento, pueden suponer que tanto su estructura como su identidad se pongan a prueba, es decir, por ejemplo, que hablamos del posible sentido utilitarista de la educación universitaria, la desafección hacia la educación ética, moral y humanística, o el desgaste de lo que se entiende por educación universitaria (Esteban, 2018).

Así, al encontrarnos en tiempos complejos llenos de incertidumbres, puede afirmarse que los valores universitarios siguen teniendo vigencia, pero los retos que se espera sean enfrentados por parte de la Universidad, suponen además grandes oportunidades de revisión con miras al futuro de la institución propiamente dicha (López Segrera, 2018). Es decir, "la Universidad tiene adelante una encrucijada: o decide romper con sus propias fronteras o no servirá para seguir venciendo nuevas fronteras del conocimiento" (Buarque, 2005, p. 9).

Primeramente, el concepto de Universidad actual se ubica básicamente en aquella institución que se organiza en función de la docencia, la investigación, la gestión, la extensión y la internacionalización (Echevarri, 2005; Mignaqui, y Lacabana, 2017; Salinas y Marín, 2017). Además, "es una institución social enmarcada en el contexto de una formación social históricamente determinada" (Malagón, 2006, p. 38; Muñoz, 2005). Como consecuencia, se infiere que, en efecto, la idea que podamos asumir como Universidad termina dibujando, en este caso, la propia idea sobre la docencia en este sector educativo (Trillo Alonso et al., 2018), encontrando esta docencia en una compleja situación que progresivamente aumenta el nivel de exigencias (Walker, 2016).

Claro está, no se debe dejar de lado que no todas las universidades tienen como punto fuerte de su propia definición, por ejemplo, el ejercicio de la investigación. Esta idea es la que más se ha popularizado a nivel global y casi se da por sentado que, en este caso, la investigación es una activi- dad propia y originaria de la Universidad, cuando lo cierto es que este aspecto se hace presente en la dinámica universitaria a partir del siglo XIX con la revolución humboldtiana (Ginés-Mora, 2018; López Segrera, 2018). Por tanto, se puede afirmar que los cimientos de la Universidad moderna se ubican a partir de la fundación de la Universidad de Berlín (1810), teniendo como máximo exponente a Humboldt. Según él, la investigación es una actividad propia de la Universidad y no de instituciones fuera de ella.

Así, si el mundo cambia permanentemente, la Universidad ha de adaptarse a esos cambios y, por ende, la idea que se tenga de ella también va a cambiar en consonancia con el contexto. Este cambio pasa de forma indiscutible por asumir que la Universidad es un órgano vivo en constante cambio y, si se quiere, en constante evolución. Por lo tanto, su razón de ser ya no será exclusivamente la de dar respuestas o soluciones a problemas de orden coyuntural, sino visualizar aquellas condiciones emergentes para las que se pueda generar un conocimiento específico (García Pereáñez, 2003; Pérez y Castaño, 2016) y, si se quiere, pasar de una estructura jerárquica a una estructura más representativa (Halffman y Radder, 2017).

No obstante, hay investigaciones que afirman que la Universidad también se resiste a los procesos de cambio (Álvarez González, 2019; Girona, Guàrdia, y Mas, 2018). Esto es compatible con la necesidad de aceptar que se requiere consolidar un modelo educativo capaz de superar la perspectiva monocultural y etnocéntrica (Rendón Uribe, 2012), con el objeto de asegurar una educación en valores y el fomento del diálogo con espíritu democrático (Tierney, 2002). De allí la importancia de que desde la propia Universidad se sienten las bases para que el posicionamiento institucional facilite el cambio y la mejora (Girona, Guàrdia, y Mas, 2018), sin dejar de reconocer que el cambio en la Universidad supone un complejo ejercicio cuando se lleva a una práctica en la que el conflicto y las interpretaciones diversas y contrapuestas también aparecen (Trillo et al., 2018). Uno de los aspectos más recurrentes es la discusión sobre la financiación de la Universidad de carácter público, lo cual incide de forma directa en su actividad y, por ende, en su actividad generadora de conocimiento (Grau y Basora, 2011). 
A juicio de Domínguez-Gómez et al. (2019), son tres los elementos que ayudan a visionar la función social de la Universidad. Esos elementos son: 1) complejidad, 2) velocidad de cambio social y 3) crisis ambiental. Entonces, la función social se erige como otro punto de atención al momento de definir lo que se entiende por Universidad, ya que esta institución se encuentra en uno de los polos de interés que tiene que ver precisamente con la correspondencia de esta con la realidad. Así, la interacción de los tres elementos ha de servir como constatación de que es la Universidad la institución que está llamada a "poner en marcha una educación ligada a la vida de la comunidad y generar procesos que influyan en el medio social a través del desarrollo científico, tecnológico, humanístico, cultural y artístico" (Duque Quintero y González Agudelo, 2009, p. 2), toda vez que se ha de reconocer que desde la Universidad se ha de fomentar la sostenibilidad y el sentido de un mundo igualitario (Alcalá del Olmo y Gutiérrez Sánchez, 2019).

Esta idea de la función social de la Universidad no se encuentra aquí de forma accidental, es decir, dadas las condiciones bajo las cuales ha vivido en estos tiempos, muchas voces críticas animan el debate político en torno a lo que se espera de ella. Este debate se nutre de aportaciones tanto de académicos como de otros actores sociales y cada uno de ellos, desde su tribuna, dirige interpelaciones importantes respecto a la acción contemporánea de la Universidad como institución social. Por otra parte, esos señalamientos tienen la particularidad de que generalmente hacen alusión a lo que fue, lo que es y lo que debería ser la Universidad de cara a un contexto que plantea una serie de "desafíos políticos, sociales, culturales, pedagógicos, comunicacionales y cognitivos" (Lion y Maggio, 2019, p. 14), toda vez que el interés por reactivar la idea de Universidad pueda servir para liberar a esa institución de la dependencia a los números, a la par de una intencionalidad que fomente la construcción de respuestas ante los desafíos como estrategia para comprender el presente (Hösle, 2018).

Ahora bien, como se ha podido perfilar en líneas anteriores, son muchos los elementos que se encuentran al momento de intentar construir un concepto o idea de lo que se entiende por Universidad. Tal y como se indicó al principio, dada la naturaleza propia de esta institución, intentar construir un único concepto es una labor difícil; no obstante, es relevante presentar en esta parte del estudio algunas constataciones de orden definitorio que sirvan como plataforma para el abordaje exploratorio, en cuanto a la dinámica seguida respecto a los cambios observados con relación a lo que se entiende por Universidad.

Se hace necesario establecer un conjunto de supuestos que influyen en los diferentes tipos de definición de Universidad y que determinan la perspectiva de su sentido. A continuación, la Tabla 1 expone cada supuesto acompañado de argumentos convergentes (a favor) y de argumentos divergentes (en contra).

En la Tabla 1 se puede identificar que la definición de Universidad es observada desde diversas perspectivas, teniendo siempre como elemento común el posible efecto sobre esta. Es decir, si se define la Universidad desde la relación Universidad-Estado-Empresa, se observa que el argumento que sirve para la concepción de Universidad está influido por la creación de mecanismos desde la sinergia entre estos tres cuerpos, mientras que la visión divergente nos recuerda, en este caso, que esos tres cuerpos persiguen fines diferentes.

Igual pasa con el resto de los supuestos, por lo cual, la revisión de esta tabla puede ayudar a dibujar las grandes tendencias que han acompañado la construcción de la definición de la Universidad. Lógicamente, ninguna visión sobre la Universidad ha estado exenta de debate y discusión, además, en todos los supuestos se considera la acción y la dinámica de la institución universitaria y cómo esta intenta adaptarse a un contexto y momento determinado.

Así las cosas, nos enfrentamos a un reto de orden disciplinario porque el ejercicio que ha de acompañar el proceso de identificación de una definición de Universidad, indiscutiblemente, implicará referentes de diversa índole. Esto obedece a que si son diferentes los supuestos que permean el intento de construcción de concepto de Universidad, igualmente serán diferentes los 
tipos de Universidad que de momento se pueden identificar. Por lo tanto, la necesidad de contar con una conceptualización o una idea que visibilice una referencia directa de lo que se entiende por Universidad, no será una labor sencilla. Esto se dificulta aún más en estos tiempos ya que, a juicio de Alfonso (2016), "la universidad hoy hace que hace. Hace que educa, cuando solo forma profesionales; hace que investiga cuando hace investigaciones que no lo son; hace que contribuye al desarrollo del país, cuando solo responde a las demandas del mercado" (p. 8).

Tabla 1 Supuestos, argumentos convergentes y divergentes influyentes en la definición de Universidad

\begin{tabular}{|c|c|c|}
\hline Supuesto & Argumentos convergentes & Argumentos divergentes \\
\hline $\begin{array}{l}\text { Desde la relación } \\
\text { Universidad- } \\
\text { Estado-Empresa }\end{array}$ & $\begin{array}{l}\text { Crear mecanismos desde la sinergia y } \\
\text { las relaciones estratégicas en pro de la } \\
\text { socialización del conocimiento. }\end{array}$ & $\begin{array}{l}\text { Estado y empresas persiguen fines di- } \\
\text { ferentes. }\end{array}$ \\
\hline $\begin{array}{l}\text { Desde la } \\
\text { tradición } \\
\text { universitaria }\end{array}$ & $\begin{array}{l}\text { Las funciones de la Universidad han } \\
\text { de conservarse, pero adaptadas al } \\
\text { contexto contemporáneo. }\end{array}$ & $\begin{array}{l}\text { La adaptación puede conllevar a la } \\
\text { mercantilización y al conocimiento } \\
\text { como bien intercambiable. }\end{array}$ \\
\hline $\begin{array}{l}\text { Desde la filosofía } \\
\text { universitaria }\end{array}$ & $\begin{array}{l}\text { Promoción del desarrollo equitativo de } \\
\text { todas las áreas del saber. } \\
\text { Asumir el pasado cultural y el valor } \\
\text { del conocimiento acumulado. } \\
\text { Libertad académica. } \\
\text { Institución que ha de superarse para } \\
\text { encontrarse. }\end{array}$ & $\begin{array}{l}\text { Docentes e investigadores en un mo- } \\
\text { delo institucional que responde más a } \\
\text { intereses empresariales o partidistas. } \\
\text { Cientificismo de excelencia. } \\
\text { Clientelismo académico. }\end{array}$ \\
\hline $\begin{array}{l}\text { Desde el modelo } \\
\text { educativo }\end{array}$ & $\begin{array}{l}\text { Pertinencia social de la formación uni- } \\
\text { versitaria. } \\
\text { Formación de ciudadanos con espíritu } \\
\text { democrático. } \\
\text { La Universidad como institución so- } \\
\text { cial. } \\
\text { Interculturalidad. } \\
\text { Estudiante como centro de atención. }\end{array}$ & $\begin{array}{l}\text { Departamentalización de las áreas. } \\
\text { Desvinculación de la formación con } \\
\text { respecto al entorno y al mundo. Inefi- } \\
\text { ciencia en la práctica de la interdisci- } \\
\text { plinariedad. } \\
\text { Actividad lectiva como centro de aten- } \\
\text { ción. }\end{array}$ \\
\hline $\begin{array}{l}\text { Desde lo } \\
\text { tecnológico }\end{array}$ & $\begin{array}{l}\text { Creación de comunidades de apren- } \\
\text { dizaje. } \\
\text { Redefinición del quehacer docente. } \\
\text { Currículum universitario integrador de } \\
\text { las TIC. }\end{array}$ & $\begin{array}{l}\text { Brecha digital. } \\
\text { Necesidad de inversión universitaria } \\
\text { - TIC. } \\
\text { Tradicionalismo institucional ante el } \\
\text { cambio. }\end{array}$ \\
\hline $\begin{array}{l}\text { Desde lo social y } \\
\text { pedagógico }\end{array}$ & $\begin{array}{l}\text { Modelo de educación intercultural e } \\
\text { integradora. } \\
\text { Universidad como síntesis de proce- } \\
\text { sos históricos, políticos, sociales, eco- } \\
\text { nómicos y culturales. }\end{array}$ & $\begin{array}{l}\text { Modelo elitista y excluyente. } \\
\text { Universidad no compleja (sin posgra- } \\
\text { do ni investigación). }\end{array}$ \\
\hline $\begin{array}{l}\text { Desde el sistema } \\
\text { educativo }\end{array}$ & $\begin{array}{l}\text { Institución integrada e integradora del } \\
\text { sistema educativo. }\end{array}$ & $\begin{array}{l}\text { Desvinculación de la Universidad con } \\
\text { el resto del sistema educativo. Au- } \\
\text { toexclusión institucional. }\end{array}$ \\
\hline $\begin{array}{l}\text { Desde la visión } \\
\text { del siglo XXI }\end{array}$ & $\begin{array}{l}\text { Universidad en conflicto cuya raíz es } \\
\text { el cambio. } \\
\text { Institución planetaria, telemática, pe- } \\
\text { dagógica, inclusiva, autónoma, tecno- } \\
\text { lógica, cuestionadora, pública, abier- } \\
\text { ta, interdisciplinaria. }\end{array}$ & $\begin{array}{l}\text { Desviación del sentido original de } \\
\text { la Universidad como institución por } \\
\text { efecto de la globalización. } \\
\text { Cambios impulsados más por moda } \\
\text { que por necesidad real. }\end{array}$ \\
\hline
\end{tabular}

Nota:

elaboración propia (2020). 
Este panorama, lejos de resultar alentador, puede significar todo un reto con una auténtica intencionalidad de cambio desde la convicción. Claro está, el hilo discursivo que se presenta en este artículo se hace desde el lado de adentro de la Universidad, sin embargo, la institución universitaria sí que debe mirar hacia afuera sin olvidar lo que es. El problema está en que no siempre se tiene la certeza de lo que es como institución. Es oportuna entonces la interrogante que a propósito de este punto expone Morin (2001, p. 1): "¿debe la Universidad adaptarse a la sociedad o la sociedad adaptarse a la Universidad?"

Parece ser que, en estos tiempos, la diversidad institucional viene siendo algo más o menos aceptado, es decir, las tipologías, sin ánimos de etiquetas poco respetuosas, ayudan a observar los objetos desde la pluralidad de escenificaciones que, en definitiva, nos pueden indicar que todos los tipos, en este caso de Universidad, efectivamente responden a una coyuntura, momento, necesidad y realidad social y política determinada. No se trata de valorar cual es la Universidad más adecuada o ideal, se trata más bien de encontrar aquel punto en el que la visión que se tiene de la institución universitaria empieza a entender que para seguir existiendo como institución, ya no resulta suficiente solo la docencia, la investigación o la gestión. Así, ya no es un secreto la gran complejidad que acompaña a los procesos de innovación y cambio en las universidades (Trillo Alonso et al., 2018), pero lo cierto es que "no se puede reformar la institución (las estructuras universitarias) si no se han reformado anteriormente las mentes: pero no se pueden reformar las mentes si no se ha reformado anteriormente la institución" (Morín, 2001, p. 4).

Una idea cercana sobre los diferentes tipos de Universidad (Tabla 2) sugiere que, ciertamente, una sola Universidad no existe, por lo tanto, del análisis que se desgrana de este aspecto resulta una posible afirmación, que en efecto la Universidad sigue teniendo influencia en los agentes sociales (Domínguez-Gómez et al., 2019). Con sus luces y sus sombras, la Universidad como institución sigue viviendo de los tiempos en que su voz era incuestionable y su prestigio social era más que evidente.
La diversidad en cuanto a los tipos de universidades trae consigo una serie de cuestionamientos que dan paso a la aparición de un nuevo interrogante: ¿Quién necesita que las universidades se diversifiquen? Es decir, la Universidad como institución social también tiene una serie de características que la pueden diferenciar, no obstante, estas características definitorias pueden producirse no por generación espontánea ni por un devenir histórico, sino por una intencionalidad concreta en su creación para satisfacer ciertas necesidades.

Ya no se trata entonces de que la Universidad se consolide como una institución que fomente cultura, valores y conocimiento (Herrán y Pesántez, 2016, p. 48). Al parecer, la excesiva departamentalización de las áreas del saber y la poca facilidad al momento de llevar a la práctica la llamada interdisciplinariedad, puede impulsar el surgimiento de instituciones universitarias que puedan a universidades independientes y que estén en la posibilidad de ofrecer formación en menos tiempo, con mayor rapidez y que en su momento sirvan para formar un talento humano requerido por un sector determinado (Sancho, Ornellas y Arrazola, 2018).

Así las cosas, pareciera que la diversificación en cuanto a los tipos de Universidad puede ser compatible con el hecho de dar respuestas a lo que imponga la dinámica de mercado (Alfonso, 2016; Girona, Guàrdia y Mas, 2018), trayendo como consecuencia que se fomente la formación de cuadros profesionales dirigida a atender una preparación que dé respuesta a una necesidad en particular sin que esto suponga un referente extrapolable a otros contextos (Brunner, 2011). Además, se reconoce que mientras se extiende la diversificación institucional, se agregan elementos de validez cuestionable (Ginés Mora, 2018) que se alejan de forma progresiva del origen en cuanto a la justificación de la existencia de varios tipos de universidades.

Ahora bien, en la Tabla 2 se observa un conjunto de universidades que se tipifican por medio de una clasificación. Esa clasificación nos permite inferir que los tipos de universidades son sumamente diversos, por lo tanto, se complica aún más el interés por definir de forma concreta el 
concepto de Universidad. Hay algunos elementos que nos permiten diferenciar a la Universidad del resto de instituciones (Acosta, 2016), pero queda demostrado que, por ejemplo, en la actualidad, existen universidades que aun siéndolo, no investigan o no tienen una departamentalización de las áreas. También existen aquellas que no forman para la transmisión de valores democráticos ni culturales, sino que, desde los programas de posgrado, persiguen formar grandes gerentes que produzcan beneficios con una formación rápida.

Tabla 2 Clasificación y tipos de Universidad

\begin{tabular}{|c|c|}
\hline Clasificación & Tipos de Universidad \\
\hline $\begin{array}{l}\text { Régimen jurídico } \\
\text { institucional }\end{array}$ & $\begin{array}{l}\text { Pública: institución controlada/administrada por un organismo público. } \\
\text { Privada: institución controlada por organizaciones no gubernamentales. } \\
\text { Privada subsidiada: institución que recibe aporte financiero regularmen- } \\
\text { te. } \\
\text { Privada independiente: institución sin apoyo financiero. }\end{array}$ \\
\hline Tipo de misión & Privada: de élite/semiélite, de identidad, no de élite/absorción de demanda. \\
\hline Tipo de función & $\begin{array}{l}\text { Universidad: de la tercera edad, de ciencias aplicadas, a distancia, abier- } \\
\text { ta, popular, tecnológica, multicultural, del trabajo, de investigación, do- } \\
\text { cente, literario-filosófica, empresarial, metafísica, burocrática, líquida, } \\
\text { cosmopolita, libidinal, auténtica, ecológica, terapéutica. }\end{array}$ \\
\hline Trayectoria histórica & $\begin{array}{l}\text { Universidades tradicionales } \\
\text { Universidades modernas (desde el siglo XIX) }\end{array}$ \\
\hline Prestigio y poder & $\begin{array}{l}\text { Universidades de clase mundial, nacionales de investigación, docentes } \\
\text { de exportación, intensivas en investigación con poco alcance transfronte- } \\
\text { rizo. Universidades eminentemente docentes. }\end{array}$ \\
\hline Exposición literaria & $\begin{array}{l}\text { Universidades napoleónicas, humboldtianas, multiversidades kerrkianas, } \\
\text { emprendedoras. }\end{array}$ \\
\hline $\begin{array}{l}\text { Influencia de } \\
\text { sucesos históricos } \\
\text { S. XX en América } \\
\text { Latina }\end{array}$ & $\begin{array}{l}\text { Universidades aristocratizantes, burguesas, torre de marfil, de abogados, } \\
\text { profesionalizantes, dependientes, comprometidas, militantes, interveni- } \\
\text { das, confesionales/aconfesionales, republicanas. }\end{array}$ \\
\hline $\begin{array}{l}\text { Porcentaje de } \\
\text { investigación }\end{array}$ & $\begin{array}{l}\text { Universidades investigadoras (publican más de } 3000 \text { artículos anuales) } \\
\text { Universidades con investigación (publican entre } 1000 \text { y } 2999 \text { artículos } \\
\text { anuales) } \\
\text { Universidades emergentes (publican entre } 50 \text { y } 200 \text { artículos anuales) } \\
\text { Universidades con actividad artesanal de investigación (publican entre } 1 \\
\text { y hasta } 49,8 \text { artículos anuales) } \\
\text { Universidades únicamente docentes (sin publicación) }\end{array}$ \\
\hline Pertinencia social & $\begin{array}{l}\text { Universidad en transición hacia la sostenibilidad. } \\
\text { Uni-pluriversidad. } \\
\text { Universidad social. } \\
\text { Universidad intercultural. }\end{array}$ \\
\hline
\end{tabular}

Nota: $\quad$ elaboración propia (2020) a partir de los datos revisados de Brunner (2011), Brunner (2012), Erdösová (2017), Malagón (2006), Murga-Menoyo (2017) y Rozo Gauta, (2001). 
Esto puede ser compatible también por el llamado efecto masificador de la educación superior. Al haber mayor masificación (Brunner, 2011), hay más necesidad de espacios formativos y, con ello, se amplía el radio de acción de la Universidad entendida como institución social. Se reconoce que la Universidad, sea cual fuere, debe ser capaz de repensar hasta qué punto su modelo educativo responde al contexto contem- poráneo en el que la práctica pedagógica exige un repensar constante y unas nuevas lógicas, por lo tanto, de cara al actual siglo XXI, la "hiperescolarización" de la educación universitaria no parece ser la medida más adecuada puesto que está inmersa en la sociedad del conocimiento en la que se cuenta, además, con un acceso irrestricto a la información.

\section{Mefodología}

Este artículo es eminentemente analítico-interpretativo (Alarcón, Múnera, y Montes, 2017) y sigue los principios de la Teoría Fundamentada a partir de dos de sus principales estrategias: el muestreo comparativo constante y el muestreo teórico. (Lúquez y Fernández, 2016). Así, desde una perspectiva hermenéutica e interpretativa se analiza un conjunto de artículos publicados en Uni-pluriversidad desde el año 2001 hasta el año 2019. Se realiza una revisión bibliográfica a la vez que se van identificando elementos simila- res o diferentes teniendo en cuenta el objeto de observación (Guirao Goris, 2015). Se revisaron 590 artículos publicados para identificar si se expresaban ideas o definiciones concretas sobre el concepto de Universidad que sirvieran como sustrato para identificar las tendencias. También se tomaron artículos que perfilaban temáticas que, aunque no estaban relacionadas directamente con el estudio de la Universidad, expresaban un tratamiento empírico sobre ella, dando como resultado final una selección de 41 artículos.

\section{Resultados y conclusiones}

Tabla 3 Elementos clave sobre la definición de Universidad expuestos en Uni-pluriversidad varios autores 2001-05

\begin{tabular}{|c|c|}
\hline $\begin{array}{l}\text { Elem e n tos } \\
\text { clave sobre la } \\
\text { definición de } \\
\text { Universidad }\end{array}$ & $\begin{array}{l}\text { Institución fundamental para el desarrollo de una nueva sociedad con la mayor } \\
\text { capacidad autorreflexiva. } \\
\text { Universidad-Estado-Empresa como relación importante. } \\
\text { La Universidad como conjunción plurivérsica. } \\
\text { Al servicio del estudiante. } \\
\text { Crítica a fragmentar el conocimiento. Compartir conocimiento nuevo y apartarse } \\
\text { de conocimientos del pasado. } \\
\text { No ha de servir ni a la empresa ni al Estado. } \\
\text { Universidad del siglo XXI en conflicto. Ha de superarse para reencontrarse. } \\
\text { Investigación con pertinencia social y científica. } \\
\text { Sentido de la Universidad: re-flexión, pre-visión y des-cubrir. } \\
\text { Internacionalización como cuarta tradición universitaria. } \\
\text { Universidad contemporánea = síntesis de procesos sociales. } \\
\text { Contextualización impuesta por la globalización y el modelo económico neoliberal. } \\
\text { Se reconcilia con su historia para recuperar aquello que aún tiene que decir. }\end{array}$ \\
\hline $\begin{array}{l}\text { Autores revi- } \\
\text { sados (2001- } \\
2005)\end{array}$ & $\begin{array}{l}\text { Alvarez (2001); Arrubla et al. (2001); Borrero (2004); Botero Bernal (2001); } \\
\text { Buarque (2005); Casanova (2005); Echevarri (2005); Echeverri y Ossa Lon- } \\
\text { doño (2001); Fischman y Stromquist (2004); García Pereáñez (2003); Garrido } \\
\text { (2002); Gil et al. (2001); Malangón Plata (2005); Morin (2001); Muñoz (2005); } \\
\text { Naidorf y Martinetto (2005); O’ Hagan (2002); Ossa Londoño y Vallejo (2003); } \\
\text { Rozo Gauta (2001); Soto Posada (2003); Tierney (2002); Vélez (2001a); Vélez } \\
\text { (2001b); Wilson y Ossa Londoño (2001); Yarce Puerta (2003). }\end{array}$ \\
\hline
\end{tabular}

Nota: $\quad$ elaboración propia (2020) a partir de los autores revisados. 
Se ha organizado la información en tres tablas para la exposición de los resultados. Cada una de ellas incluye información que se ha agrupado en tres bloques a partir de los autores revisados: de 2001 a 2005, de 2006 a 2011 y de 2012 a 2019. Ahora bien, recordemos que Uni-pluriversidad empieza su actividad en el año 2001. Los primeros cinco años de vida de la Revista fueron de una gran producción intelectual cuyo epicentro fue indudablemente la Universidad como institución. Esta producción también se centró en la capacidad autorreflexiva de la Universidad para interpretar los grandes fenómenos que ocurrían en su contexto (Tabla 3).

En estos primeros cinco años se observa que el discurso sobre la Universidad como definición gira en torno a su deber ser. Por ejemplo, si bien es cierto que se reconoce la relación Universi-
dad-Empresa-Estado, también se reconoce que cada uno de estos cuerpos tiene sus diferencias y, en el caso de la Universidad, ella no estará al servicio de los otros dos. Por otra parte, el contexto de la llegada del nuevo milenio sirvió como punto de atención para que desde Uni-pluriversidad se expusieran aquellos elementos que servirían para que la Universidad fuese una institución adaptada a un nuevo contexto, sin perder de vista su singularidad.

Para los años 2006-2011, Uni-pluriversidad adquiere un matiz social y socio-constructivo respecto a la idea de Universidad. Así, los autores que expusieron sus ideas para aquel entonces defendieron una real y auténtica pertinencia social de la Universidad, a la vez que lo plural y lo intercultural transversalizaban su definición (Tabla 4):

Tabla 4 Elementos clave sobre la definición de Universidad expuestos en Uni-pluriversidad varios autores 2006-11

\begin{tabular}{|l|l|}
\hline $\begin{array}{l}\text { Elementos clave } \\
\text { sobre la defini- } \\
\text { ción de Univer- } \\
\text { sidad }\end{array}$ & $\begin{array}{l}\text { Pertinencia social de la universidad, acción educativa vinculada a la comunidad. } \\
\text { Institución corporativa, universal, científica, autónoma. } \\
\end{array}$ \\
& $\begin{array}{l}\text { Funciones. Investigación, enseñanza, servicio al hombre y a la sociedad. } \\
\text { Transformación socioeconómica del país. } \\
\text { Construye diversidad, pluralidad, diálogo intercultural, respeto ambiental. } \\
\text { Problemas al enfrentar la práctica de la interdisciplinariedad desde la universi- } \\
\text { dad. } \\
\text { Particularidades en su origen fomentan prácticas institucionales inadecuadas. } \\
\text { Necesidad de asumir y consolidar la condición de universidad social. } \\
\text { Universidad pública que forma talento humano y profesionales. } \\
\text { Institución de libertad, con espíritu pensante. } \\
\text { Institución que actúa con la fuerza del conocimiento. } \\
\text { Genera procesos influyentes en el medio social desde desarrollo científico, tec- } \\
\text { nológico, humanístico, cultural y artístico. } \\
\text { Libertad académica. }\end{array}$ \\
\hline $\begin{array}{l}\text { Autores revi- } \\
\text { sados (2006- } \\
\text { 2011) }\end{array}$ & $\begin{array}{l}\text { Barragán (2010); Borrero (2007); Cifuentes Bejarano (2007); Duque Quintero } \\
\text { yoda (2006); Rendón Uribe (2010); Restrepo, Franco y Quiroz (2011); Zapata } \\
\text { Sasco (2008). }\end{array}$ \\
\hline
\end{tabular}

Nota:

elaboración propia (2020) a partir de los autores revisados.

Tal y como se infiere de la tabla anterior, en este período resalta el elemento social de la Universidad. Si bien es cierto que se reconocen las funciones de la institución universitaria, también es cierto que desde Uni-pluriversidad se expusie- ron una serie de líneas definitorias respecto a la Universidad que giraron en torno al rol que esta debe cumplir tanto para la comunidad, como para el desarrollo económico del país. Tiene presente que la institución ha de generar una serie 
de procesos que han de influir en todas las esferas y sectores sociales, para lo cual, la libertad académica era parte fundamental y lógicamente poder superar los inconvenientes al momento de llevar a la práctica la interdisciplinariedad.

Para los años 2012-2019, Uni-pluriversidad da un paso más en cuanto a su idea de Universidad a través de los autores que para entonces ex- pusieron sus investigaciones. Mientras que entre 2001-2005 el reconocimiento de la Universidad como institución era el aspecto central y entre 2006-2011 la pertinencia social fue el denominador más común, durante los años 2012-2019 se expusieron trabajos académicos que coincidían en la consolidación de la Universidad como institución y su trascendencia (Tabla 5).

Tabla 5 Elementos clave sobre la definición de Universidad expuestos en Uni-pluriversidad varios autores 2012-2019

\begin{tabular}{|l|l|}
\hline $\begin{array}{l}\text { Elementos clave } \\
\text { sobre la definición } \\
\text { de Universidad }\end{array}$ & $\begin{array}{l}\text { Conocimiento acumulado, talento humano - infraestructura, capacidad de } \\
\text { socializar e intercambiar conocimiento como principal fortaleza de la uni- } \\
\text { versidad. } \\
\text { Modelo educativo universitario que supere la perspectiva tradicional, mono- } \\
\text { cultural y etnocéntrica. } \\
\text { Fomento de la educación intercultural. } \\
\text { Innovación, cambio en el quehacer docente, compartir conocimientos, co- } \\
\text { munidades de aprendizaje, construcción del conocimiento. } \\
\text { Universidad intercultural que enseña un contexto étnicamente complejo. } \\
\text { Interacción cotidiana con personas de diferente contexto sociocultural. } \\
\text { “Universitología" como campo para entender y estudiar la universidad como } \\
\text { fenómeno sociocultural y su diversidad organizativa. } \\
\text { Universidad como epicentro de iniciativas reformistas. } \\
\text { Institución de natural contradicción. } \\
\text { Institución de pensamiento, debate, pluralidad y transmisión de valores y } \\
\text { cultura. }\end{array}$ \\
\hline Eutores revisados \\
Lozano (2012); Ruiz y Duarte (2017); Toledo Lara (2019).
\end{tabular}

Nota:

elaboración propia (2020) a partir de los autores revisados.

Se infiere que la universidad empieza a verse como aquella institución que no solo investiga o forma a ciudadanos; da un paso más allá en cuanto a su rol en un mundo cada vez más convulso. La Universidad, haciendo uso del conocimiento que ha podido compilar a través de los años, ha de observar su entorno y desarrollar un modelo educativo que supere prácticas formativas tradicionales para transitar hacia un modelo que apueste por la innovación, la interculturalidad y las comunidades de aprendizaje sin dejar de lado que siempre ha de balancearse entre la autocrítica y la contradicción como una de sus estrategias para la generación y socialización de conocimiento.
Además, en este periodo se destaca el aporte inédito de esta revista al estudio de la Universidad como fenómeno sociocultural, exponiendo en el mundo académico lo que se conoce como "Universitología" (Ruiz y Duarte, 2017). Esta se entiende como iniciativa para analizar y comprender el surgimiento de la Universidad como fenómeno desde el ámbito sociocultural, su dinámica y cómo ha acompañado el devenir de la sociedad sin dejar de reconocer sus luces y sus sombras ya que, la Universidad no pasa desapercibida y es allí cuando se defiende la necesidad de su estudio.

Ahora bien, en todos los textos revisados y analizados se ha observado que no existe una 
comprensión homogénea de Universidad. Existen elementos comunes que se asocian con el rol de esta institución, pero lo que más variación tiene es precisamente lo que ella debe ser y, curiosamente, el aspecto común es que la Universidad no existe para ella misma, sino que su razón de ser reposa en la medida en que existe para los demás. Es decir, la pertinencia social de la Universidad no está en entredicho, pero se cuestiona la forma en la que se ha entendido esa pertinencia social.

Cada una de las concepciones de Universidad expuestas desde la revista Uni-pluriversidad está en sintonía con el momento y el contexto, sin perder la visión de lo que será en un futuro, pero con la crítica permanente a lo que se hace desde el presente. Es oportuno rescatar la visión que expuso Rozo Gauta (2001) sobre el interés de participar en Uni-pluriversidad, justamente en el año en que esta revista inició su andadura:

Invitamos a hablar de la UNI-versidad que tenemos, de la PLURI-versidad que deseamos y soñamos, de la conjunción plurivérsica de los conocimientos y de los sujetos - hoy todavía fragmentados - que administramos, enseñamos y somos, y de esa necesidad que hoy compartimos con otros científicos y profesores del mundo (p. 5).

La cita anterior se puede asumir como la céIula primigenia que ha servido para establecer la identidad de la idea de Uni-pluriversidad; en la cual, un pensamiento único se concibe como una limitación a la de conocimiento. En este caso, el debate académico casado con el análisis de la realidad se erige como sustrato de primer orden al momento de sentar las bases de una especie de encrucijada de saberes que desde Uni-pluriversidad se quiere impulsar. En ese orden de ideas, la revista Uni-pluriversidad se ha convertido a lo largo de estos años en uno de los pocos espacios académicos pensados principalmente para el análisis e implicaciones de la Universidad.

En consecuencia, se asume que la Universidad sigue siendo un referente social de progreso (Acosta, 2016; Pérez y Castaño, 2016), que no está separada de los procesos de transformación integral y global (Murga-Menoyo, 2017) y, aun- que muestre obsesión por los rankings e impactos (Ginés-Mora, 2018), se empeña en transmitir y socializar conocimiento desde un sentido educativo integral (Pérez y Castaño, 2016). Claro está, no se deja de aceptar que, como institución humana (Ginés-Mora, 2018), el sentido de crisis ha estado presente en ella y, con esto, los intentos reformistas desde hace más de treinta años (Hösle, 2018) permiten asumir como punto de discusión, si lo que está haciendo con la educación es realmente correcto, considerando todas las alternativas y posibilidades (Esteban, 2018).

La crítica, la investigación con pertinencia social y científica, la docencia desde la ética y la transmisión de valores culturales, ciudadanos y democráticos no son elementos ajenos a la Universidad contemporánea (Ginés Mora, 2018; López Segrera, 2018; Ossa Londoño y Vallejo, 2003; Pérez y Castaño, 2016) con lo cual, ni con las prácticas más innovadoras ni con los diseños institucionales más de vanguardia, la Universidad no dejaría de ser una gran referencia social. En definitiva, "como quizás a ninguna otra institución, le pertenece a ella la capacidad crítica" (Hösle, 2018, p. 2).

El replanteamiento de la Universidad como concepto pasa por reconocer que es una institución en permanente crisis. Esa crisis es su forma de vida ya que así, ella misma se reinventa, recrea y refunda a partir de los heterogéneos y profundos fenómenos sociales (Alfonso, 2016; Hösle, 2018; Vélez, 2001). Con ello se acepta que "los momentos de crisis producen escenarios para nuevas tomas de decisiones y ponen en evidencia problemas que permanecían ocultos a ojos de la mayor parte de la ciudadanía" (Porlán, 2020, p. 1).

Este sentido pluralista y plurivérsico también ha significado que diferentes posturas y puntos de vista, tanto convergentes como divergentes, tengan su espacio de socialización en la revista. Precisamente, la diversidad de posturas y puntos de vista hacen de Uni-pluriversidad, un referente para que muchos académicos de diferentes latitudes encuentren en esta revista el espacio para contrastar ideas y compartir el conocimiento científico. La existencia de grandes trasnacionales dedicadas a la comercialización del saber no 
ha sido impedimento para que Uni-pluriversidad, poco a poco, se consolide como una revista cuyo aval principal sea, por una parte, veinte años de existencia y, por otra, erigirse como la síntesis del conocimiento desde diversas formas de acceder a él, superando el tradicionalismo y extremismo disciplinario entre lo cuantitativo y lo cualitativo, dando así la oportunidad de la reconciliación interdisciplinaria e inter-metodológica.

Uni-pluriversidad tiene en sus manos el reto de continuar siendo una tribuna en la que se expongan estudios que permitan observar la forma en que la Universidad se entiende. Además, Uni-pluriversidad ha de reconocerse como una de las pocas revistas que tiene como epicentro la Universidad como institución ya que, mientras se observan otras revistas que perfilan su quehacer desde la educación superior, esta enfoca su generación de conocimiento en y desde la Universidad y cómo esta arropa una dinámica en permanente cambio. Este fenómeno es un punto de interés que se ha venido consolidando a lo largo de 20 años.

De cara a la mejora de los procesos asociados a la internacionalización de la investigación desde Uni-pluriversidad, la difusión, tanto de los estudios que se realizan en esta revista como las futuras estrategias comunicacionales, genera un mayor intercambio de saberes y conocimientos por parte de los investigadores de otras partes del mundo. Se hace necesario, por ejemplo, fomentar números temáticos sobre temas específicos de Universidad, tales como: políticas públicas universitarias, internacionalización de la Universidad, lo público y lo privado en la Universidad, la Universidad actual y la tradición universitaria, entre otros. En síntesis, la Universidad como punto de interés investigativo siempre será una gran oportunidad para generar conocimiento y transmitir valores y cultura.

\section{Referencias}

Acosta, J. (2016). Interdisciplinariedad y transdisciplinariedad: perspectivas para la concepción de la universidad por venir. Alteridad, 11(1), 148156.

Alarcón, A., Munera, L., y Montes, A. (2017). La teoría fundamentada en el marco de la investigación educativa. Saber, ciencia y libertad, 12(1), 236245.

Alcalá del Olmo, M., y Gutiérrez Sánchez, J. (2019). El desarrollo sostenible como reto pedagógico de la universidad del siglo XXI. Anduli: Revista Andaluza de Ciencias Sociales, 19, 59-80.

Alfonso, J. (2016). La universidad hoy. LÍMITE Revista Interdisciplinaria de Filosofía y Psicología, 11(37), 7-20.

Álvarez González, F. J. (2019). Grandes desafíos actuales de la innovación pedagógica en la Educación Superior. En Universidad Nacional de Educación del Ecuador (Ed.), UNAE Las Raíces del cambio. Libro de rendición de cuentas de la Universidad Nacional de Educación de Ecuador, (pp.2-30). Dirección Editorial UNAE.

Alvarez, M. (2001). Reingeniería del aula de clase para los procesos de enseñanza-aprendizaje de investigación y de producción de conocimiento propio. Uni-pluriversidad, 1(3), 9-10.
Arrubla, J. P., Oquendo, S., Preciado, J. M., y Ossa Londoño, J. (2001). Factores clave de éxito de los grupos y centros de investigación de excelencia y consolidados de la Universidad de Antioquia. Uni-pluriversidad, 1(1), 17-23.

Barragán, B. (2010). La Enseñanza en la Universidad: interdisciplinariedad, cultura académica y experiencia. Uni-pluriversidad, 10(2), 36-43.

Borrero, A. (2004). Pasado, presente y futuro de la Universidad Colombiana. Uni-pluriversidad, 4(3), 19-25.

Borrero, A. (2007). Resumen ejecutivo del libro La Universidad como una Institución hoy. Uni-pluriversidad, 7(3), 71-78.

Botero Bernal, A. (2001). Diagnóstico filosófico de los paradigmas de enseñanza universitaria. Uni-p/uriversidad, 1(2), 29-38.

Brunner, J. (2011). Universidad para todos. Revista Estudios Públicos, 124, 151-170.

Brunner, J. (2012). La universidad: ¿comunidad de mercado o posmoderna? Bordón. Revista de Pedagogía, 64(3), 12. 27-38.

Buarque, C. (2005). Universidad sin fronteras. Uni-pluriversidad, 5(2), 9-12. 
Casanova, H. (2005). La Universidad: Tensiones y futuro. Uni-pluriversidad, 5(3), 11-17.

Cifuentes Bejarano, N. (2007). La Universidad ¿Qué es? Autonomía y Educación para lo Superior. Uni-pluriversidad, 7(3), 25-32.

Domínguez-Gómez, J., Pinto, H., y González-Gómez, T. (2019). La función social de la Universidad hoy: del prestigio institucional al posicionamiento ético. Oficina do CES, (453), 1-26.

Duque Quintero, S., y González Agudelo, E. (2009). De la memorización y aplicación de la norma a la popularización del derecho en la educación jurídica clínica: un reto para la didáctica universitaria. Uni-pluriversidad, 9(1), 1-11.

Echevarri, J. (2005). La educación superior en la coyuntura actual. Uni-pluriversidad, 5(1), 59-66.

Echeverri, J. V., y Ossa Londoño, J. (2001). La universidad: entre la regionalización y la globalización. Uni-pluriversidad, 1(2), 19-24.

Erdösová, Z. (2017). Problemática sociolingüística de las universidades interculturales mexicanas a la luz de su ideología educativa. Uni-pluriversidad, 17(1), 59-71.

Esteban, F. (2018). Ideas de ayer para la Educación Universitaria de hoy. Foro de Educación, 16(24), 215-232.

Fischman, G., y Stromquist, N. (2004). Impacto de la globalización en las universidades de los países del tercer mundo. Uni-pluriversidad, 4(1), 4156.

García Pereáñez, A. (2003). Acercamiento a la delimitación de una problemática: sobre el deber ser de la educación superior. Uni-pluriversidad, 3(3), 43-52.

Garrido, M. (2002). El nuevo contrato entre ciencia y sociedad. Uni-pluriversidad, 2(3), 9-11.

Gil, D., Gallego, H., Rozo, J., Zapata, J., Alzate, L., Rugeles, M., Cadavid, A., Durango, O., Echavarría, H., Echeverry, V., y Ossa Londoño, J. (2001). Reflexiones sobre el proceso de admisión de la Universidad de Antioquia, a propósito de una experiencia de intervención social. Uni-pluriversidad, 1(1), 25-28.

Ginés Mora, J. (2018). Universidades: mitos, modas y tendencias. Revista iberoamericana de educación superior, 9(24), 3-16.

Girona, C., Guàrdia, L., y Mas, X. (2018). La docencia universitaria más allá del 2020: tendencias, retos y nuevos escenarios. En S. Carrasco y I. De Corral (Coords.) Docencia universitaria e innovación, (pp. 195-226). Octaedro.
Grau, F. X., y Basora, D. (2016). La universidad pública de hoy: dimensión, eficacia y eficiencia. $L a$ Cuestión Universitaria, (7), 4-15.

Guirao Goris, S. (2015). Utilidad y tipos de revisión de literatura. Ene, 9(2), 1-17. https://dx.doi. org/10.4321/S1988-348X2015000200002

Halffman, W. y Radder, H. (2017). Manifiesto académico: de la universidad ocupada a la universidad pública. Cuadernos de Información y Comunicación, 22, 259-281.

Herrán, J., y Pesántez, A. (2016). La universidad innovadora. Revista cubana de educación superior, 35(3), 47-63.

Hösle, V. (2018). La idea de universidad ante los desafíos del siglo XXI. Documentos Core Currículum, 8, 1-22.

Lion, C., y Maggio, M. (2019). Desafíos para la enseñanza universitaria en los escenarios digitales contemporáneos. Aportes desde la investigación. Cuadernos de Investigación Educativa, 10(1), 13-25. https://dx.doi.org/10.18861/ cied.2019.10.1.2878

López Segrera, F. (2018). Retos de la educación superior en un contexto de incertidumbre y crisis global. Avaliação: Revista da Avaliação da Educação Superior (Campinas), 23(2), 551-566.

Lúquez, P., y Fernández, O. (2016). La teoría fundamentada: precisiones epistemológicas, teórico-conceptuales, metodológicas y aportes a las ciencias. Cumbres, 2(1), 101-114.

Malagón, L. (2006). Perspectiva economicista en la vinculación universidad-sociedad. Uni-pluriversidad, 6(2), 37-45.

Malangón Plata, L. (2005). Cambios y Conflictos en los Discursos Político-pedagógicos sobre la Universidad. Uni-pluriversidad, 5(1), 83-96.

Mignaqui, V., y Lacabana, M. (2017). Los retos del desarrollo sostenible para las universidades. Integración y conocimiento, 6(2), 256-271.

Montoya de la Cruz, G. (2011). Universidad pública: sobre la pertinencia e impertinencia de lo social como horizonte. Uni-pluriversidad, 11(2), 151155.

Morin, E. (2001). De la reforma universitaria. Uni-pluriversidad, 1(2), 74-79.

Muñoz, D. (2005). De cómo la idea de universidad de Karl Jaspers preexistía en la América Latina del siglo XIX y principios del XX. Uni-pluriversidad, 5 (2), 2-13.

Murga-Menoyo, M. (2017). Universidades en transición. Hacia una transformación institucional 
orientada al logro de la sostenibilidad. Revista Iberoamericana de Educación, 73, 61-84.

Naidorf, J., y Martinetto, A. (2005). Los límites a la autonomía universitaria en el marco del debate sobre el desarrollo científico-tecnológico. Uni-pluriversidad, 5(1), 25-38.

O'Hagan, C. (2002). Las universidades globales: ¿Sembrando las semillas del futuro o ancladas en el pasado? Uni-pluriversidad, 2 (2), 51-56.

Ossa Londoño, J., y Vallejo, M. (2003). Sueños de universidad. Uni-pluriversidad, 3(1), 76-77.

Pérez, S., y Castaño, R. (2016). Funciones de la Universidad en el siglo XXI: humanística, básica e integral. Revista electrónica interuniversitaria de formación del profesorado, 19(1), 191-199.

Porlán, R. (2020). El cambio de la enseñanza y el aprendizaje en tiempos de pandemia. Revista de Educación Ambiental y Sostenibilidad 2(1), 1502. doi: $10.25267 /$ Rev educ ambient sostenibilidad.2020.v2.i1.1502

Posada, S. (2006). La formación del universitario: un gran desafío para la universidad. Uni-pluriversidad, 6(2), 1-7.

Pulgarín Silva, R. (2013). El reto de innovar en el proceso docente universitario. Uni-pluriversidad, 13(1), 13-15.

Rendón Uribe, M. (2010). Los estilos de enseñanza en la Universidad de Antioquia (Primera etapa Facultad de Educación). Uni-pluriversidad, 10(1), 5-22.

Rendón Uribe, M. (2012). Las convivencias y las competencias ciudadanas en la universidad. Uni-pluri/versidad, 12 (2), 57-72

Restrepo, R., Franco, N., y Quiroz, R. (2011). Educación Superior e Imaginarios de Género. Uni-pluriversidad, 11(1), 16-23.

Rodríguez Lozano, M. (2012). La dicotomía entre la investigación básica y la investigación aplicada y sus implicaciones en el campo de la educación. Uni-pluriversidad, 12(1), 11-13.

Rozo Gauta, J. (2001). UNI-PLURI/VERSIDAD. Uni-pluriversidad, 1(1), 5-6.

Ruiz, H., y Duarte, F. (2017). Un ejercicio de ruptura en la construcción del currículo en la Educación Superior. Caso Universidad Piloto de Colombia. Uni-pluriversidad, 17(2), 88-101.

Salinas, J., y Marín, V. (2017). La universidad entre lo real y lo virtual: una trayectoria no lineal para la didáctica universitaria. Notandum, 44-45.
Sancho, J., Ornellas, A., y Arrazola, J. (2018). La situación cambiante de la universidad en la era digital. RIED. Revista Iberoamericana de Educación a Distancia, 21, (2), 31-49.

Soto Posada, G. (2003). La Humanitas como Universitas en el medioevo. Uni-pluriversidad, 3(3), 11-22.

Tierney, W. (2002). La universidad después del 11 de septiembre. Uni-pluriversidad, 2(2), 6-10.

Toledo Lara, G. (2019). De Bolonia a España: constataciones y consideraciones sobre la reforma universitaria. Uni-pluriversidad, 19(1), 14-32.

Trillo Alonso, F., Zabalza Beraza, M., y Zabalza Cerdeiriña, M. (2018). ¿Hemos cambiado? La perspectiva de los profesores eméritos sobre el cambio educativo en la universidad. Educatio sig/o XXI, 36 (2), 255-276.

Velázquez Jiménez, C. (2016). La formación en investigación de los docentes universitarios. Estudio de caso en una Institución de Educación Superior Colombiana. Uni-pluriversidad, 16(1), 15-25.

Vélez, H. (2001a). La Universidad: "ente" histórico fluido. Uni-pluriversidad, 1(2), 39-44.

Vélez, H. (2001b). Universidad, Empresa, Estado: Encuentros y desencuentros. A propósito de las alianzas estratégicas. Uni-pluriversidad, 1(2), 5-7.

Walker, S. (2016). El trabajo docente en la universidad: condiciones, dimensiones y tensiones. Perfiles educativos, 38(153), 105-119.

Wilson Osorio, J., y Ossa Londoño, J. (2001). Notas para la historia de la universidad colombiana, al cierre del siglo XX. Uni-pluriversidad, 1(2), 9-18.

Yarce Puerta, R. (2003). La Universidad: Academia vs. Capitalismo. Uni-pluriversidad, 3(2), 11-15.

Zapata Vasco, J. (2008). La educación universitaria y la formación humanística: Un reto por construir. Uni-pluriversidad, 8 (3), 1-11. 